\title{
Detection of Visual Activation in the Rat Brain Using 2-deoxy-2-[18 F]fluoro-D-glucose and Statistical Parametric Mapping (SPM)
}

\author{
M. L. Soto-Montenegro, ${ }^{1}$ J. J. Vaquero, ${ }^{1}$ J. Pascau, ${ }^{1}$ J. D. Gispert, ${ }^{2}$ P. García-Barreno, ${ }^{1}$ \\ M. Desco ${ }^{1,3}$ \\ ${ }^{1}$ Unidad de Medicina y Cirugia Experimental, Hospital General Universitario Gregorio Marañón, C/Dr. Esquerdo, 46, Madrid 28007, Spain \\ ${ }^{2}$ Institut d'Alta Tecnologia PRBB. CRC Corporació Sanitària, Dr, Aiguader, 88, Barcelona 08003, Spain \\ ${ }^{3}$ Centro de Investigacion Biomedica en Red de Salud Mental, CIBERSAM, C/Dr. Esquerdo, 46, Madrid 28007, Spain
}

\begin{abstract}
Purpose: This study was designed to assess changes in brain glucose metabolism in rats after visual stimulation.

Materials and methods: We sought to determine whether visual activation in the rat brain could be detected using a small-animal positron emission tomography (PET) scanner and 2-deoxy-2$\left[{ }^{18} \mathrm{~F}\right]$ fluoro-D-glucose (FDG). Eleven rats were divided into two groups: (a) five animals exposed to ambient light and (b) six animals stimulated by stroboscopic light $(10 \mathrm{~Hz})$ with one eye covered. Rats were injected with FDG and, after $45 \mathrm{~min}$ of visual stimulation, were sacrificed and scanned for $90 \mathrm{~min}$ in a dedicated PET tomograph. Images were reconstructed by a threedimensional ordered subset expectation maximization algorithm $(1.8 \mathrm{~mm}$ full width at half maximum). A region-of-interest (ROI) analysis was performed on 14 brain structures drawn on coronal sections. Statistical parametric mapping (SPM) adapted for small animals was also carried out. Additionally, the brains of three rats were sliced into $20-\mu \mathrm{m}$ sections for autoradiography.

Results: Analysis of $\mathrm{ROI}$ data revealed significant differences between groups in the right superior colliculus, right thalamus, and brainstem $(p \leq 0.05)$. SPM detected the same areas as the ROI approach. Autoradiographs confirmed the existence of hyperactivation in the left superior colliculus and auditory cortex.

Conclusions: To our knowledge, this is the first report that uses FDG-PET and SPM analysis to show changes in rat brain glucose metabolism after a visual stimulus.
\end{abstract}

Key words: PET, FDG, Cerebral glucose metabolism, Rats, SPM

\section{Introduction}

Q everal imaging techniques for conducting brain activa$\checkmark$ tion studies are currently available to the experimental neuroscientist. Positron emission tomography (PET) enables quantitative in vivo determinations of various physiological and biochemical processes. The tracer 2-deoxy-2- $\left[{ }^{18} \mathrm{~F}\right]$ fluoro-D-glucose (FDG) is commonly used with PET to image normal and altered metabolic states in several disease processes in humans and small experimental animals.

For brain activation assessment, it is assumed that changes in FDG uptake reflect an increased metabolic demand following changes in neuron firing. Light-induced functional changes in neural activity eventually affect the glucose metabolism of cortical and subcortical structures. Thus, regional changes in brain FDG uptake can indirectly reflect changes in brain function associated with cognitive or behavioral tasks. 
The primary visual pathway in albino mammals is characterized by lower percentages of ipsilateral retinofugal fibers and enhanced innervation of contralateral thalamic targets [1-6]. Because of the greater predominance of contralateral projections in these albino animals, monocular stimulation (left eye obscured) is predicted to produce less activation in the visual system structures contralateral to the obscured eye (the right) than in those ipsilateral to the obscured eye, for those parts of the visual system that are predominantly monocular (the superior colliculus and the thalamus), but not in those parts that are predominantly binocular (visual cortex).

Sensitivity to light stimulation in albino and pigmented rodents has been studied using behavioral and electrophysiological techniques [7-12]; however, there are no studies using in vivo imaging techniques.

In our study, the left eye cover also obscured the left ear. Previous work in mammals [13, 14] has shown that auditory thalamic and sensory cortex respond more strongly to contralateral than to ipsilateral stimulation. Thus, obscuring the left ear should result in less stimulation to auditory thalamic and cortical structures on the right side of the brain as compared to the left side of the brain in strobe-lightstimulated animals.

The goal of our study was to assess the feasibility of detecting changes in brain glucose metabolism in albino rats after a visual stimulus, by using FDG and a small-animal PET scanner.

\section{Materials and Methods}

\section{Subjects}

Wistar female rats weighing 260 to $300 \mathrm{~g}$ were maintained in cages in a room with a $12 \mathrm{~h}$ light dark cycle and fed with commercial rodent laboratory chow and water ad libitum. The animals were deprived of food but allowed free access to water for $10 \mathrm{~h}$ before the PET scan. All animal procedures were in compliance with the European Communities Council Directive of 24 November 1986 (86/609/EEC) and were approved by the Institutional Animal Care and Use Committee of our Hospital.

\section{Study Design}

The animals were anesthetized with isoflurane $(5 \%$ for induction and $11.5 \%$ for maintenance in $100 \% \mathrm{O}_{2}$ ). A polyethylene catheter was inserted into a femoral vein to facilitate injection of FDG. The catheter was buried under the skin in a tunnel running from the femoral area to the back of the neck, thus allowing the animal to move freely without damaging the catheter. After catheterization, surgical wounds were sutured and treated with 5\% lidocaine ointment. Body temperature was monitored by a rectal probe and maintained at $37^{\circ} \mathrm{C}$ by a thermostatically controlled heating lamp. Duration of anesthesia during the surgical preparation was usually about $30 \mathrm{~min}$. At least $3 \mathrm{~h}$ were allowed for recovery from the surgery and anesthesia before the animal underwent visual stimulation and PET imaging.
The animals were divided into two groups:

Group A $(N=5)$ : animals maintained in ambient light Group B $(N=6)$ : animals stimulated with stroboscopic light pulsing at $10 \mathrm{~Hz}$ with the left eye and ear covered

The animals were then positioned in front of the stroboscopic lamp and restrained with a loose fitting plaster cast around the pelvis, which allowed free movement of the upper body. The animals were not under anesthesia during the $45 \mathrm{~min}$ of ambient or stroboscopic light exposure. The stroboscopic device used in this study to deliver visual stimuli produced not only light flashes but also produced audible click sounds when switching on the light.

\section{FDG-PET Study}

2 deoxy $2\left[{ }^{18} \mathrm{~F}\right]$ fluoro $D$ glucose $(2.18 \pm 0.25 \mathrm{mCi})$ was injected through the intravenous catheter. The animals underwent light stimulation for $45 \mathrm{~min}$ and were sacrificed with a bolus venous injection of Pentothal (Abbott Laboratories) solution before being imaged for $90 \mathrm{~min}$.

Imaging was performed with a piPET scanner [15]. This system has two detectors $18 \mathrm{~cm}$ apart, which consisted of arrays of $26 \times 22$ BGO crystals $(2 \times 2 \times 10 \mathrm{~mm})$ coupled to a position sensitive photomultiplier tube.

Tomographic images were reconstructed using a three dimen sional ordered subset expectation maximization algorithm (20 iterations, five subsets) [16], creating 43 tomographic images of $102 \times 102$ pixels that spanned the $55 \times 45 \mathrm{~mm}$ field of view of the scanner. The reconstructed voxel size was $0.55 \times 0.55 \times 1.1 \mathrm{~mm}^{3}$, and the spatial resolution in these images was $1.65 \mathrm{~mm}$ full width at half maximum (FWHM) isotropic. The energy window was 300 $650 \mathrm{keV}$, and decay and dead time corrections were applied. The usefulness of the piPET imaging device has been reported elsewhere [17].

Fourteen regions of interest (ROIs) of at least 10 pixels $\left(3.3275 \mathrm{~mm}^{3}\right)$ were drawn on coronal views of different areas of the visual and auditory systems: left superior colliculus (1 SC), right superior colliculus (r SC), left visual cortex (1 VC), right visual cortex ( $\mathrm{r}$ VC), left thalamus (l th), right thalamus ( $\mathrm{r}$ th), left somatosensory cortex (1 SSC), right somatosensory cortex ( $r$ $\mathrm{SSC}$ ), brain stem (BS; from the boundary with the spinal cord up to the thalamus, superior colliculus excluded), left amygdala (1 AA), right amygdala (r AA), right auditory cortex (r AC), left auditory cortex (1 AC), and whole brain (WB). Because of the limited anatomical resolution of the PET images, ROIs were positioned by identifying the $3 \mathrm{D}$ coordinates of each structure in a rat brain atlas [18].

A background ROI was also drawn outside the brain region. The activity of this ROI was very low in all the studies, indicating a small contribution from random coincidence events and from scatter. To normalize FDG uptake, tissue activity was corrected by subtracting background and dividing by whole brain activity. The results are expressed as a percentage (\%) according to the expression:

$$
\text { FDG uptake }=\left[\begin{array}{c}
\text { (study ROI activity background activity }) \\
\text { whole brain activity }
\end{array}\right]
$$$$
\times 100
$$ 


\section{Autoradiography}

To further validate the PET results, after the FDG PET scan, the brains of two rats from group $\mathrm{B}$ and one from group A were extracted, frozen in isopentane at $40^{\circ} \mathrm{C}$, sliced in a cryostat at $20^{\circ} \mathrm{C}$ into $20 \mu \mathrm{m}$ coronal sections, dried on glass slides, and exposed for $12 \mathrm{~h}$ at room temperature for FDG autoradiography. Images were obtained on a Fujifilm BAS 1500 scanner. Autoradio grams were visually examined to verify areas with high uptake of FDG in the PET images.

\section{Data Analysis}

ROI Data Statistics ROI based data were analyzed using one way analysis of variance (ANOVA). Data normality and homoscedasticity were previously assessed by the Kolmogorov Smirnov test and the Levene test.

Statistical Parametric Mapping Rigid body transformations should be enough to register rat brains since their anatomy is quite similar provided the animals are within a certain weight range [19]. In our study, we created a template by registering and averaging all the images from group A to a single reference image. The registration algorithm was based on the maximization of mutual information [20, 21]. Finally, the whole dataset (groups A and B) was then registered to this template. The results were checked visually and no misregistration was detected.

A brain mask was then manually segmented on the template and applied to all registered scans before intensity normalization. The resulting images were smoothed with a Gaussian kernel $(1.0 \times 1.0 \times$ $2.0 \mathrm{~mm}$ FWHM) and analyzed with the SPM2b software package (SPM, Wellcome Department of Imaging Neuroscience, Institute of Neurology, UCL, London, UK) [22], using an ANOVA design to detect differences between groups. Results are provided for two thresholds of uncorrected $p$ values $(<0.05$ and $<0.01)$, both for a minimum cluster size of ten adjacent voxels.

\section{Results}

\section{PET Study}

The ANOVA analysis revealed significant differences between groups in the right superior colliculus, right thalamus, right auditory cortex, and brainstem $(p \leq 0.05)$. These structures showed lower FDG uptake in those rats stimulated with stroboscopic light. The analysis also revealed significantly smaller activation in the right superior colliculus when both hemispheres were compared $(p \leq 0.05)$.

Figure 1 illustrates these results with coronal views of the PET scan of one rat from group B (left panel), where the lower FDG uptake in the right superior colliculus can be observed. The right panel shows the autoradiography at the same level.

Table 1 summarizes the effect of light stimulation on brain glucose metabolism in the different ROIs measured.

SPM analysis detected no significant increases in FDG between groups. However, it did find significant decreases in FDG uptake in the brainstem (14\%), right superior colliculus $(12 \%)$, and right $(7 \%)$ and left thalamus $(5 \% ; p<0.01 ; t=$
$2.28 ; k=10$ voxels). Figure 2 shows the statistical parametric map for decreases in FDG uptake after visual stimulus for $p<$ 0.01 . For the $p$ value threshold $p<0.05 \quad(t=1.83)$, a new structure appeared, the right auditory cortex $(9 \%)$. Figure 3 shows the statistical parametric map for decreases in FDG uptake after visual stimulus for $p<0.05$.

Table 2 summarizes the effect of light stimulation on brain glucose metabolism by SPM analysis.

\section{Autoradiographic Study}

Visual inspection of the autoradiographs showed notably smaller activation in the left superior colliculus compared to the right side (Fig. 1). No other area showed visually salient changes in brain glucose metabolism. Quantification was not performed. The results obtained by autoradiography were consistent with those of both the ROI- and SPM-based approaches and with those of previous reports.

\section{Discussion}

To our knowledge, this is the first report that uses a smallanimal PET scanner and SPM analysis to show changes in brain glucose metabolism in rats undergoing visual stimulation (ambient vs. flashing light).

For the experiment, we used albino rats because of their higher light sensitivity and more rapid signal transmission at lower light levels [10]. Moreover, albino specimens show a larger crossed pathway than uncrossed pathway, thus favoring a better visualization of the visual pathway by FDG-PET imaging [23-25].

Conventionally, PET images are analyzed manually by drawing ROIs. This technique is time-consuming, as the complete study must be segmented on a slice-by-slice basis and subjective error when defining the ROIs may introduce inaccuracies in the image analysis. Furthermore, ROI-based analyses are usually hypothesis-driven, in such a way that possible significant effects outside hypothesized ROIs may be missed. The use of SPM analysis can alleviate these

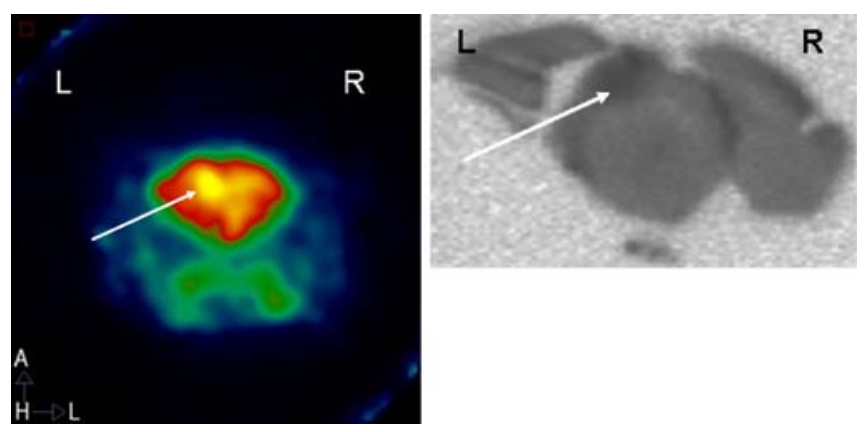

Fig. 1. Coronal brain slices from a rat that was stimulated at $10 \mathrm{~Hz}$. The lower uptake of FDG in right superior colliculus is noticeable (left panel, FGD image; right panel, autoradiograph). 
Table 1. ROI analysis: tissue activity has been normalized to whole-brain activity and results are shown as a percentage

\begin{tabular}{|c|c|c|c|}
\hline & \multicolumn{2}{|l|}{ Visual paradigm } & \multirow[t]{2}{*}{$p$} \\
\hline & Control ( $n$ 5 5$)$ & Stimulated at $10 \mathrm{~Hz}(n \quad 6)$ & \\
\hline Right superior colliculus & $144.21 \pm 5.51$ & $130.84 \pm 3.27 *, * *(13 \%)$ & 0.05 \\
\hline Left superior colliculus & $141.99 \pm 3.42$ & $146.16 \pm 5.34$ & 0.55 \\
\hline Right visual cortex & $105.10 \pm 4.05$ & $101.81 \pm 2.79$ & 0.51 \\
\hline Left visual cortex & $107.03 \pm 2.36$ & $104.56 \pm 2.93$ & 0.54 \\
\hline Right thalamus & $122.89 \pm 3.04$ & $114.52 \pm 1.48 *(8 \%)$ & 0.03 \\
\hline Left thalamus & $120.89 \pm 1.63$ & $117.27 \pm 2.11$ & 0.22 \\
\hline Right somatosensory cortex & $104.69 \pm 3.60$ & $101.87 \pm 2.75$ & 0.54 \\
\hline Left somatosensory cortex & $104.23 \pm 1.51$ & $104.23 \pm 3.58$ & 1.00 \\
\hline Brainstem & $122.48 \pm 3.95$ & $110.97 \pm 2.51 *(11 \%)$ & 0.03 \\
\hline Right amygdala & $89.68 \pm 5.31$ & $90.54 \pm 4.07$ & 0.89 \\
\hline Left amygdala & $91.89 \pm 4.91$ & $93.33 \pm 3.90$ & 0.82 \\
\hline Right auditory cortex & $115.04 \pm 4.3$ & $104.49 \pm 9.17 *(9 \%)$ & 0.04 \\
\hline Left auditory cortex & $108.29 \pm 7.68$ & $111.75 \pm 7.46$ & 0.47 \\
\hline
\end{tabular}

Values expressed as mean $\pm \mathrm{SD}$

$* p<0.05$ versus control group; $* * p<0.05$ versus interhemispheric

drawbacks. In humans, this technique is the method of choice for the statistical analysis of brain functional data obtained using PET, single-photon emission computed tomography, and functional magnetic resonance imaging (fMRI) [26-29]. SPM has been used for many years in clinical research to detect subtle activity changes in the brain and is an excellent exploratory tool, as it does not require a priori assumptions about the expected brain region activations.

The SPM technique had to be adapted so that it could be used for rat brain studies. Few studies have used SPM techniques in rats with different imaging modalities: autoradiographic iodoantipyrine [30-34], fMRI [35-40], and FDGPET [20, 41, 42]. Two PET studies used a standardization according to a custom digital template matched to a highresolution microMRI image in Paxinos orientation [41, 42]. The third study analyzed FDG-PET images by comparing different registration approaches to align small-animal PET studies. It also proposed a procedure to validate the results by means of objective measurements of registration consistency that would enable SPM to be applied to small-animal brains $[20]$.

In our study, the SPM technique detected significant regional brain activations that overlapped most regions that were also detected with a standard ROI analysis. Activations appeared in visual circuits (superior colliculus, thalamus), auditory circuits (auditory cortex), and in motor areas (brainstem). This result is consistent with the nature of the task. Furthermore, it also agrees with the metabolic changes in different brain regions of the rat reported by Toga and Collins [43], who studied the visual system in albino rats using the 2-deoxy-D- $\left[{ }^{14} \mathrm{C}\right]$-glucose quantitative autoradiography method.

We did not find changes in any structures related to stress. The possible effects of immobilization stress on the local cerebral metabolic rate of glucose has been investigated elsewhere, and no significant differences were found either $[44,45]$.
In the subcortex, SPM detected several activation regions that were also found on the ROI analysis. These included the right superior colliculus and right thalamus, which contains the lateral geniculate nucleus. It is well known that the superficial layers of the superior colliculus in the rat are the major targets of retinal inputs [46] and respond to pulsed visual stimuli $[47,48]$. Therefore, afferents from the superior colliculus project to the visual thalamus. Toga et al. [44, 45] found that the metabolic changes were greatest in the superior colliculus, less so in the lateral geniculate, and considerably less in second-order sites such as layer IV of the visual cortex. Moreover, these changes were frequency dependent in the superior colliculus and lateral geniculate,

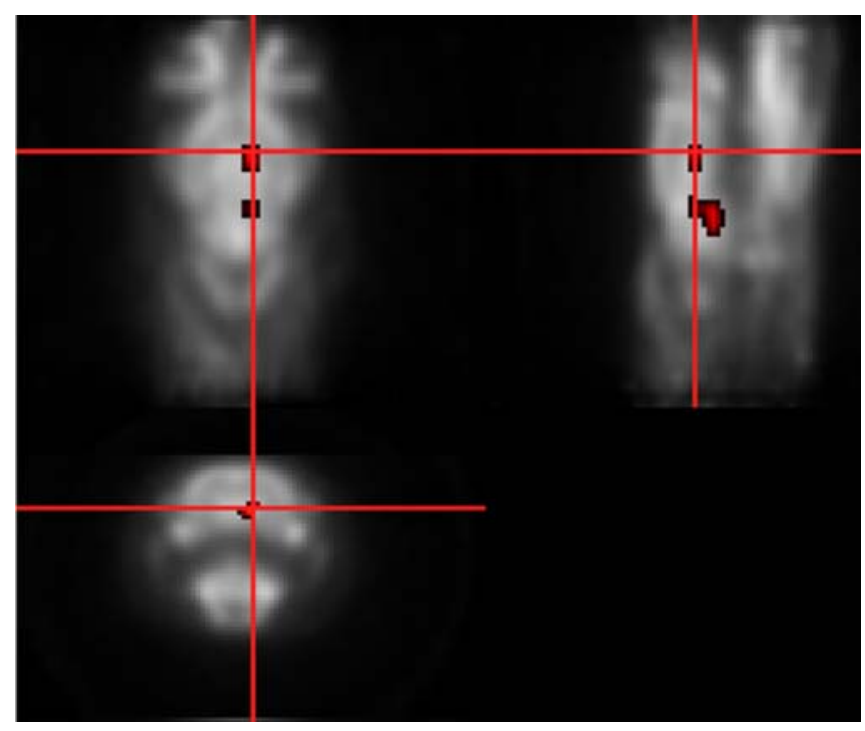

Fig. 2. Statistical parametric map for decreases in FDG uptake after visual stimulus. Color scale represents all $T$ distributions achieving statistical significance $(p=0.01, k=10$ voxels). 


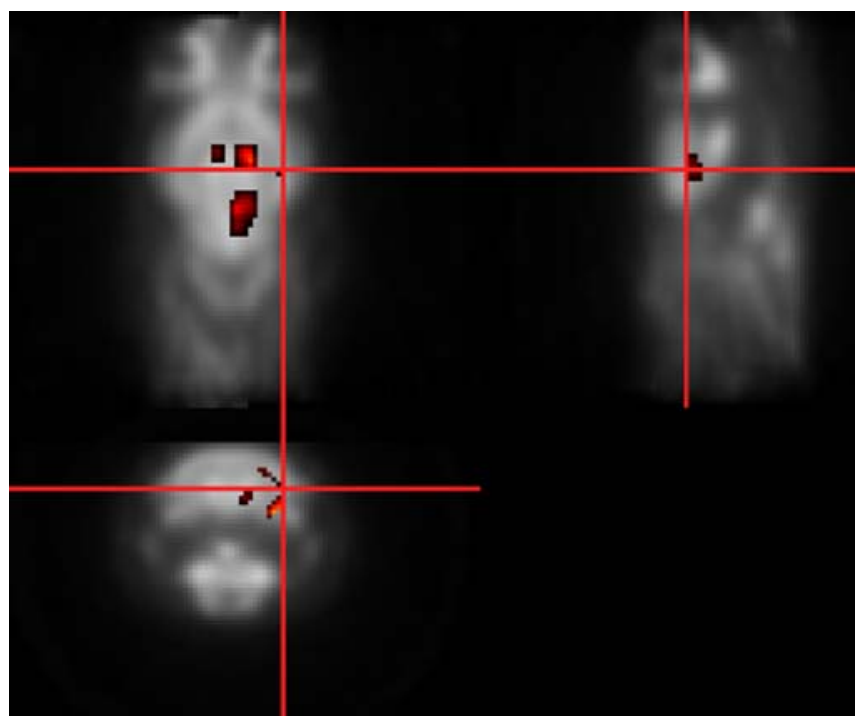

Fig. 3. Statistical parametric map for decreases in FDG uptake after visual stimulus. Color scale represents all $\mathrm{T}$ distributions achieving statistical significance $(p=0.05, k=10$ voxels).

showing a greater metabolic rate at higher frequencies of stimulation. In our study, we used only one frequency $(10 \mathrm{~Hz})$, and we observed significant changes in the superior colliculus and thalamus.

Unexpectedly, both SPM and ROI analysis detected hypoactivation in the brainstem and right auditory cortex. A possible explanation for detecting activation in right auditory cortex is that the stroboscopic device used in our study produced a noise that was synchronous with light flashes, thus producing a concomitant auditory stimulus. Many auditory areas of the brainstem are binaural, so if one ear is covered, there may not be an overall difference in metabolic response- - however, the inferior colliculus would show a difference in activation $[13,49]$ that could make the overall levels lower in the strobe condition. Moreover, it is well known that the brainstem is related to the basic motor and somatic sensory pathway, where there is a wide variety of sensorial nuclei (cochlear, vestibular, pedunculopontine tegmental). In fact, the changes in FDG uptake in the brainstem may be explained by the fact that not only was the left eye covered but the left ear was also covered. This means that there may have been a lower input from these sensorial nuclei in the brainstem and, consequently, less FDG uptake in that area.

In our study, the SPM analysis confirmed the pattern of predicted right-left differences in activation for the visual and auditory components of the strobe-stimulated animalslower activation on the right side in the thalamus and auditory cortex for auditory stimulation and lower activation on the right side in the superior colliculus and thalamus but not in the visual cortex for visual stimulation. The ROI analysis, however, only found some of these differences.
SPM detected one additional unpredicted activation region, the left thalamus, that did not appear in the ROI analysis. We found lower FDG uptake in this region, but we cannot offer a sound explanation for this fact. The thalamus is known to have multiple functions since it acts as a translator for various "prethalamic" inputs from the auditory, somatic, visceral, gustatory, and visual systems. The size and shape of this region when defining the ROI and the number of slices on which it appears may have prevented ROI assessment from finding it. Moreover, if any region of brain activation is smaller than the defined ROI, significant activations may be missed. Because significance in SPM is established at a voxel level, it theoretically allows for more accurate detection of activations. In the case of complex paradigms, SPM could detect functional brain activations, which are difficult to predict a priori, as reported elsewhere [30].

Our study has several limitations. The first is the low anatomical resolution of the PET images. Measurements of metabolic activity by this technique in small regions may not be entirely accurate since ROI activity could be contaminated by that of surrounding brain regions. This is also the reason why the brainstem was studied as a single region, not dividing into left and right side. In future studies, metabolic activity could be improved if partial volume effects were corrected by defining the ROI on registered magnetic resonance imaging (MRI) scans of the same animal. SPM can take advantage of this MRI scan if the template used is an MRI image instead of a PET image. Second, the sample size was relatively small. Nevertheless, the number of animals in each group proved sufficient to detect significant changes in glucose metabolism.

In conclusion, this is the first report to show changes in rat brain glucose metabolism using FDG-PET after a visual stimulus. Our data suggest that a small-animal PET scanner could be suitable for studies of cognitive or behavioral tasks.

Acknowledgments. This work was supported by grants from the Ministerio de Ciencia y Tecnología (TEC2004-07052), Ministerio de Sanidad y

Table 2. SPM analysis: left side shows the effect size for the $p$ value threshold $p<0.01$ and the right side shows the effect size for he $p$ value threshold $p<0.05$

\begin{tabular}{lll}
\hline & \multicolumn{2}{c}{ Visual paradigm } \\
\cline { 2 - 3 } & SPM $(p<0.01)$ & SPM $(p<0.05)$ \\
\hline $\begin{array}{l}\text { Right superior colliculus } \\
\text { Left superior colliculus }\end{array}$ & $\downarrow 12 \%$ & $\downarrow 12 \%$ \\
$\begin{array}{l}\text { Right visual cortex } \\
\text { Left visual cortex }\end{array}$ & \\
$\begin{array}{l}\text { Right thalamus } \\
\text { Left thalamus }\end{array}$ & $\downarrow 7 \%$ & \\
$\begin{array}{l}\text { Right somatosensory cortex } \\
\text { Left somatosensory cortex }\end{array}$ & $\downarrow 5 \%$ & $\downarrow 7 \%$ \\
$\begin{array}{l}\text { Brainstem } \\
\text { Right amygdala }\end{array}$ & $\downarrow 14 \%$ & $\downarrow 14 \%$ \\
$\begin{array}{l}\text { Left amygdala } \\
\text { Right auditory cortex }\end{array}$ & & \\
\begin{tabular}{l} 
Left auditory cortex \\
\hline
\end{tabular} & & $\downarrow 9 \%$ \\
\hline
\end{tabular}


Consumo (CIBERsam CB07/09/0031 and Plan Nacional sobre Drogas 2007/043), Ministerio de Industria (CDTEAM Project), and Fundación de Investigación Médica Mutua Madrileña. We thank Alexandra de Francisco for her assistance with the PET studies, the Atomic, Molecular, and Nuclear Physics Department of the Universidad Complutense in Madrid for reconstructing the PET images, and the National Institute of Health for facilitating the piPET system.

\section{References}

1. Lund RD, Lund JS (1965) The visual system of the mole Talpa europaea. Exp Neurol 13(3):302 316

2. Lund RD, Lund JS, Wise RP (1974) The organization of the retinal projection to the dorsal lateral geniculate nucleus in pigmented and albino rats. J Comp Neurol 158(4):383 403

3. Guillery RW (1971) An abnormal retinogeniculate projection in the albino ferret (Mustela furo). Brain Res 33(2):482 485

4. Guillery RW (1974) Visual pathways in albinos. Sci Am 230(5):44 54

5. Guillery RW (1996) Why do albinos and other hypopigmented mutants lack normal binocular vision, and what else is abnormal in their central visual pathways. Eye 10(Pt 2):217 221

6. Klooster J, van der Want JJ, Vrensen G (1983) Retinopretectal projections in albino and pigmented rabbits: an autoradiographic study. Brain Res 288(1 2):1 12

7. Balkema GW (1988) Elevated dark-adapted thresholds in albino rodents. Invest Ophthalmol Vis Sci 29(4):544 549

8. Balkema GW, Drager UC (1991) Impaired visual thresholds in hypopigmented animals. Vis Neurosci 6(6):577 585

9. Balkema GW, MacDonald S (1998) Increased absolute light sensitivity in Himalayan mice with cold-induced ocular pigmentation. Vis Neurosci 15(5):841 849

10. Herreros de Tejada P, Green DG, Munoz Tedo C (1992) Visual thresholds in albino and pigmented rats. Vis Neurosci 9(3 4):409 414

11. Munoz Tedo C, Herreros de Tejada P, Green DG (1994) Behavioral estimates of absolute threshold in rat. Vis Neurosci 11(6):1077 1082

12. Naarendorp F, Sato Y, Cajdric A et al (2001) Absolute and relative sensitivity of the scotopic system of rat: electroretinography and behavior. Vis Neurosci 18(4):641 656

13. Fitzpatrick DC, Batra R, Stanford TR et al (1997) A neuronal population code for sound localization. Nature 388(6645):871 874

14. King AJ, Bajo VM, Bizley JK et al (2007) Physiological and behavioral studies of spatial coding in the auditory cortex. Hear Res 229(1 2):106 115

15. Siegel S, Vaquero JJ, Aloj L et al (1999) Initial results from a PET/ Planar small animal imaging system. IEEE Trans on Nuclear Sci 46 (3):571 575

16. Herráiz JL, España S, Vaquero JJ et al (2006) FIRST: Fast Iterative Reconstruction Software for (PET) tomography. Phys Med Biol $51: 45474565$

17. Jagoda EM, Vaquero JJ, Seidel J et al (2004) Experiment assessment of mass effects in the rat: implications for small animal PET imaging. Nucl Med Biol 31(6):771 779

18. Kruguer L, Saporta S, Swanson LW (1995) Photographic atlas of the rat brain: the cell and fiber architecture illustrated in three planes with stereotaxic coordinates. Cambridge University Press, New York, p 299

19. Rubins DJ, Melega WP, Lacan G et al (2003) Development and evaluation of an automated atlas-based image analysis method for microPET studies of the rat brain. Neuroimage 20(4):2100 2118

20. Pascau J, Gispert JD, Michaelides M et al (2008) Automated method for small-animal PET image registration with intrinsic validation. Mol Imaging Biol. doi:10.1007/s11307-008-0166-Z

21. Pluim JP, Maintz JB, Viergever MA (2003) Mutual-information-based registration of medical images: a survey. IEEE Trans Med Imaging 22 (8):986 1004

22. Friston KJ, Holmes AP, Worsley KJ et al (1995) Statistical parametric maps in functional imaging: a general linear approach. Hum Brain Mapp 2:189 210

23. Dorey SE, Neveu MM, Burton LC et al (2003) The clinical features of albinism and their correlation with visual evoked potentials. Br J Ophthalmol 87(6):767 772

24. Neveu MM, Jeffery G, Burton LC et al (2003) Age-related changes in the dynamics of human albino visual pathways. Eur J Neurosci 18 (7):1939 1949
25. Ilia M, Jeffery G (2000) Retinal cell addition and rod production depend on early stages of ocular melanin synthesis. J Comp Neurol 420(4):437 444

26. Friston KJ, Frith CD, Liddle PF et al (1991) Comparing functional (PET) images: the assessment of significant change. J Cereb Blood Flow Metab 11(4):690 699

27. Turner R, Howseman A, Rees GE et al (1998) Functional magnetic resonance imaging of the human brain: data acquisition and analysis. Exp Brain Res 123(1 2):5 12

28. Ito K, Morrish PK, Rakshi JS et al (1999) Statistical parametric mapping with ${ }^{18} \mathrm{~F}$-dopa PET shows bilaterally reduced striatal and nigral dopaminergic function in early Parkinson's disease. J Neurol Neurosurg Psychiatry 66(6):754 758

29. Kiebel SJ, Friston KJ (2004) Statistical parametric mapping for eventrelated potentials: I. Generic considerations. Neuroimage 22(2):492 502

30. Nguyen PT, Holschneider DP, Maarek JM et al (2004) Statistical parametric mapping applied to an autoradiographic study of cerebral activation during treadmill walking in rats. Neuroimage 23(1):252 259

31. Holschneider DP, Yang J, Sadler TR et al (2006) Mapping cerebral blood flow changes during auditory-cued conditioned fear in the nontethered, nonrestrained rat. 29:1344 1358

32. Lee JS, Ahn SH, Lee DS et al (2005) Voxel-based statistical analysis of cerebral glucose metabolism in the rat cortical deafness model by 3D reconstruction of brain from autoradiographic images. Eur J Nucl Med Mol Imaging 32(6):696 701

33. Yang J, Sadler TR, Givrad TK et al (2007) Changes in brain functional activation during resting and locomotor states after unilateral nigrostriatal damage in rats. Neuroimage 36(3):755 773

34. Holschneider DP, Yang J, Guo Y et al (2007) Reorganization of functional brain maps after exercise training: importance of cerebellar thalamic cortical pathway. Brain Res 1184:96 107

35. Cross DJ, Flexman JA, Anzai Y et al (2008) Age-related decrease in axonal transport measured by MR imaging in vivo. Neuroimage 39 (3):915 926

36. Easton N, Marshall F, Fone KC et al (2007) Differential effects of the D-and L-isomers of amphetamine on pharmacological MRI BOLD contrast in the rat. Psychopharmacology (Berl) 193(1):11 30

37. Easton N, Marshall F, Fone K et al (2007) Atomoxetine produces changes in cortico-basal thalamic loop circuits: assessed by phMRI BOLD contrast. Neuropharmacology 52(3):812 826

38. Littlewood CL, Jones N, O'Neill MJ et al (2006) Mapping the central effects of ketamine in the rat using pharmacological MRI. Psychopharmacology (Berl) 186(1):64 81

39. Lowe AS, Williams SC, Symms MR et al (2002) Functional magnetic resonance neuroimaging of drug dependence: naloxone-precipitated morphine withdrawal. Neuroimage 17(2):902 910

40. Schweinhardt P, Fransson P, Olson L et al (2003) A template for spatial normalisation of MR images of the rat brain. J Neurosci Methods 129 (2):105 113

41. Casteels C, Vermaelen P, Nuyts J et al (2006) Construction and evaluation of multitracer small-animal PET probabilistic atlases for voxel-based functional mapping of the rat brain. J Nucl Med 47(11):1858 1866

42. van Kuyck K, Casteels C, Vermaelen P et al (2007) Motor-and food-related metabolic cerebral changes in the activity-based rat model for anorexia nervosa: a voxel-based microPET study. Neuroimage 35(1):214 221

43. Toga AW, Collins RC (1981) Metabolic response to optic centers to visual stimuli in the albino rat: anatomical and physiological considerations. J Comp Neurol 199(4):443 464

44. Soncrant TT, Holloway HW, Stipetic M et al (1988) Cerebral glucose utilization in rats is not altered by hind limb restraint or by femoral artery and vein cannulation. J Cereb Blood Flow Metab 8(5):720 726

45. Crane AM, Porrino LJ (1989) Adaptation of the quantitative $2-\left[{ }^{14} \mathrm{C}\right]$ deoxyglucose method for use in freely moving rats. Brain Res 499(1):87 92

46. Linden R, Perry VH (1983) Retrograde and anterograde-transneuronal degeneration in the parabigeminal nucleus following tectal lesions in developing rats. J Comp Neurol 218(3):270 281

47. Cooper RM, Thurlow GA (1991) [2-14C]deoxyglucose uptake in rat visual system during flashing-diffuse and flashing-pattern stimulation over a 6 log range of luminance. Exp Neurol 113(1):79 84

48. Cooper RM, Allen K (1995) Metabolic activity in rat visual system during exposure to high and low intensities of patterned and diffuse light. Int J Neurosci 81(1 2):27 34

49. Thompson SK, von Kriegstein K, Deane-Pratt A et al (2006) Representation of interaural time delay in the human auditory midbrain. Nat Neurosci 9(9):1096 1098 